\title{
Effects of pressure on the magnetic-structural and Griffiths-like transitions in $\mathrm{Dy}_{5} \mathrm{Si}_{3} \mathrm{Ge}$
}

\author{
N. Marcano, ${ }^{1,2,3}$ P. A. Algarabel,,${ }^{2,3}$ J. Rodríguez Fernández, ${ }^{4}$ C. Magén, ${ }^{3,5,6}$ L. Morellón,,${ }^{3,5}$ Niraj K. Singh, ${ }^{7}$ \\ K. A. Gschneidner, Jr., ${ }^{7,8}$ V. K. Pecharsky, ${ }^{7}$ and M. R. Ibarra ${ }^{1,3,5}$ \\ ${ }^{1}$ Centro Universitario de la Defensa, Academia General Militar Crta. Huesca s/n 50090 Zaragoza, Spain \\ ${ }^{2}$ Instituto de Ciencia de Materiales de Aragón, CSIC-Universidad de Zaragoza, 50009 Zaragoza, Spain \\ ${ }^{3}$ Departamento Física de la Materia Condensada, Universidad de Zaragoza, 50009 Zaragoza, Spain \\ ${ }^{4}$ Departamento CITIMAC, Universidad de Cantabria, 39005 Santander, Spain \\ ${ }^{5}$ Instituto de Nanociencia de Aragón, Universidad de Zaragoza, 50018 Zaragoza, Spain \\ ${ }^{6}$ Fundación ARAID, 50018 Zaragoza, Spain \\ ${ }^{7}$ Ames Laboratory, U.S. Department of Energy, Iowa State University, Ames, Iowa 50011-3020, USA \\ ${ }^{8}$ Department of Materials Science and Engineering, Iowa State University, Ames, Iowa, 50011-2030, USA \\ (Received 3 June 2013; revised manuscript received 2 October 2013; published 30 December 2013)
}

\begin{abstract}
Magnetization studies have been performed on a polycrystalline sample of $\mathrm{Dy}_{5} \mathrm{Si}_{3} \mathrm{Ge}$ as a function of an applied magnetic field (up to $50 \mathrm{kOe}$ ) and hydrostatic pressure (up to $10 \mathrm{kbar}$ ) in the 5-300 $\mathrm{K}$ temperature range. The anomalous behavior of the magnetic susceptibility indicates that a Griffiths-like phase exists at low magnetic fields and pressures up to $10 \mathrm{kbar}$. We present evidence that the high-temperature second-order ferromagnetic transition can be coupled with the low-temperature first-order crystallographic transformation into a single first-order magnetic-crystallographic transformation using a magnetic field and hydrostatic pressure as tuning parameters. The effect of pressure on the Griffiths-like phase is reported and analyzed in the framework of the complex competition between the interslab and intraslab magnetic interactions.
\end{abstract}

DOI: 10.1103/PhysRevB.88.214429

PACS number(s): 75.30.Kz, 75.50.Cc, 62.50.-p, 75.30.Sg

\section{INTRODUCTION}

Since the discovery of the giant magnetocaloric effect (GMCE) near the magnetostructural transition in $\mathrm{Gd}_{5} \mathrm{Si}_{2} \mathrm{Ge}_{2}{ }^{1,2}$ there has been a growing interest in $R_{5} T_{4}(R=$ rare earth; $T=\mathrm{Si}$ and $\mathrm{Ge}$ ) compounds and their solid solutions. Many of these alloys show a strong magnetostructural coupling manifested in the occurrence of structural and magnetic transitions that can be reversibly induced by modifying one or more external parameters such as magnetic field, temperature, or hydrostatic pressure. As a consequence, remarkable physical properties have been observed in this family of compounds including giant magnetoresistance, ${ }^{3}$ giant magnetostriction, ${ }^{4}$ and giant magnetocaloric effect. ${ }^{1}$

The observed phenomenology of the 5:4 family is associated with a peculiar crystal structure, which is intrinsically layered, and is built by stacking of stable two-dimensional subnanometer-thick layers (slabs) of $R$ and $\mathrm{Si} / \mathrm{Ge}$ atoms ${ }^{5}$ and the strong interplay between the magnetic and structural degrees of freedom. The crystallographic phase and the nature of the magnetic interactions are controlled by the number of interlayer covalentlike $(\mathrm{Si} / \mathrm{Ge})-(\mathrm{Si} / \mathrm{Ge})$ bonds connecting the slabs. ${ }^{6}$ The rearrangement of these slabs via changing specific $(\mathrm{Si} / \mathrm{Ge})-(\mathrm{Si} / \mathrm{Ge})$ bonds gives rise to different crystal structures adopted by this family of materials. Compounds with all the slabs connected through strong $(\mathrm{Si} / \mathrm{Ge})-(\mathrm{Si} / \mathrm{Ge})$ bonds adopt the $\mathrm{Gd}_{5} \mathrm{Si}_{4}$-type orthorhombic structure [also referred to as the $O$ (I) state with space group Pnma], whereas those without any interslab $(\mathrm{Si} / \mathrm{Ge})-(\mathrm{Si} / \mathrm{Ge})$ bonding possess the $\mathrm{Sm}_{5} \mathrm{Ge}_{4}$-type structure [referred to as the $O$ (II) structure, space group Pnma]. The compounds with alternate slabs connected through the $(\mathrm{Si} / \mathrm{Ge})-(\mathrm{Si} / \mathrm{Ge})$ bonds belong to the $\mathrm{Gd}_{5} \mathrm{Si}_{2} \mathrm{Ge}_{2}$-type monoclinic crystal structure ( $M$ state, space group $\left.P 112_{1} / a\right)$.
Recent studies reveal the presence of Griffiths-like phases (GP) in several compounds of the $R_{5}\left(\mathrm{Si}_{x} \mathrm{Ge}_{1-x}\right)_{4}$ family ( $R=$ $\mathrm{Gd}, \mathrm{Tb}, \mathrm{Dy}$, and Ho) adopting the $M$ and $O(\mathrm{II})$ structural phases at room temperature. ${ }^{7,8}$ The appearance of this regime originates from the local disorder within the crystallographic structure, stabilized and enhanced by the competing intralayer and interlayer magnetic interactions. Hydrostatic pressure allows one to control the bonding and distance between the slabs, being a key parameter to determine the crystallographic and magnetic states in these alloys. The extraordinary sensitivity of the electronic and crystal structures to the application of hydrostatic pressure have led to a systematic investigation over the last few years (see review in Ref. 9) in order to more completely comprehend the microscopic processes taking place in the 5:4 materials. Among all of them, $\mathrm{Er}_{5} \mathrm{Si}_{4}$ shows the most outstanding behavior under hydrostatic pressure. In this compound the structural and magnetic transitions are reported to be unusually far apart, i.e., they are separated by a temperature difference of $\sim 200 \mathrm{~K}$. On cooling, this system undergoes a first-order crystallographic phase transition $O(I) \rightarrow M$ in the paramagnetic (PM) state at $T_{t} \sim 200-230 \mathrm{~K}^{10,11}$ and becomes ferromagnetic (FM) at low temperature, $T_{C}=30 \mathrm{~K} \cdot{ }^{10,12-14}$ Hydrostatic pressure not only induces the $O(\mathrm{I})$ phase at low temperature, but also shifts the high-temperature crystallographic change at a very high rate of $d T_{t} / d P \sim-30 \mathrm{~K} / \mathrm{kbar}^{15,16}$ This causes both transitions (the high-temperature crystallographic and the low-temperature magnetic ordering) to collapse at high pressures (above $6 \mathrm{kbar}$ ), which stabilizes the $O(\mathrm{I})-\mathrm{Er}_{5} \mathrm{Si}_{4}$ over the whole temperature range maximizing the magnetocaloric effect at low temperature. ${ }^{17}$

$\mathrm{Tb}_{5} \mathrm{Si}_{2} \mathrm{Ge}_{2}$ is another example that exhibits weakly decoupled magnetic and structural transitions. The structural change occurs approximately $10 \mathrm{~K}$ below $T_{C}=110 \mathrm{~K}$ at 
ambient pressure. ${ }^{18}$ Hydrostatic pressure has been shown to be a tool producing a recoupling of both the magnetic and crystallographic transitions because the structural transition temperature and $T_{C}$ exhibit different pressure dependences (the rate of change of the former is nearly one order of magnitude greater than that of the latter). This difference in the rate results in a spectacular enhancement of the MCE in this compound when both transitions are recoupled. ${ }^{19}$

The extent of decoupling of structural and magnetic transitions in $\mathrm{Dy}_{5} \mathrm{Si}_{3} \mathrm{Ge}$ places it halfway between $\mathrm{Tb}_{5} \mathrm{Si}_{2} \mathrm{Ge}_{2}$ and $\mathrm{Er}_{5} \mathrm{Si}_{4}$. Previous studies on $\mathrm{Dy}_{5} \mathrm{Si}_{3} \mathrm{Ge}$ revealed that it has the monoclinic $M$-type structure at room temperature..$^{20}$ Magnetization measurements indicated that it orders ferromagnetically at $T_{C} \sim 65 \mathrm{~K}$, which is preceded by an antiferromagnetic (AFM) transition. On cooling, an incomplete structural transformation from $M$ to the $O(\mathrm{I})$ phase takes place at $T_{t} \sim 50 \mathrm{~K}^{21}$ The closely spaced ferromagnetic and structural transitions lead to a hysteresis in the magnetization data as expected for a first-order phase transition. ${ }^{21}$ Below $\sim 50 \mathrm{~K}, \mathrm{Dy}_{5} \mathrm{Si}_{3} \mathrm{Ge}$ exists in a structurally phase-separated state and is ferromagnetically ordered. It is noteworthy that the structural transition is incomplete at the lowest recorded temperature $(\sim 47 \%$ vol. of the $O$ (I) phase is observed at $T=5 \mathrm{~K}^{21}$ ). Moreover, a GP phase behavior above the AFM state was suggested. Recently, it was proposed that $\mathrm{Ge}$ and $\mathrm{Si}$ atoms preferentially occupy the intraslab and interslab sites, respectively, which leads to dominant FM interactions within both the orthorhombic and monoclinic structures. ${ }^{22}$

Application of hydrostatic pressure in $\mathrm{Dy}_{5} \mathrm{Si}_{3} \mathrm{Ge}$ should lead to a moderate increase of the second-order Curie temperature $T_{C}$ and Néel temperatures $T_{N}$ whereas a much stronger effect is expected at the first-order structural transformation $T_{t}$. Thus, it is reasonable to investigate whether the application of pressure results in a recoupling of the anomalies in this compound. The aim of this work is to investigate the magnetic and structural phase diagrams of $\mathrm{Dy}_{5} \mathrm{Si}_{3} \mathrm{Ge}$ under hydrostatic pressure. We have performed a complete study of the magnetic properties by measuring the magnetization up to an applied magnetic field of $50 \mathrm{kOe}$, as a function of temperature and hydrostatic pressure. From these measurements the different pressure-magnetic-field-temperature phase diagrams have been determined. The anomalous behavior of the magnetic susceptibility indicates the existence of a GP at low applied magnetic fields and applied hydrostatic pressures up to $10 \mathrm{kbar}$. The effect of hydrostatic pressure on GP phase is presented and discussed in the framework of the complex competition between the interslab and intraslab magnetic interactions.

\section{EXPERIMENT}

A polycrystalline $\mathrm{Dy}_{5} \mathrm{Si}_{3} \mathrm{Ge}$ sample was prepared by arc melting of pure constituent elements taken in stoichiometric proportions under inert argon atmosphere. Dy was prepared by the Materials Preparation Center at the Ames Laboratory ${ }^{23}$ and it was 99.9 at.\% pure with respect to all other elements in the periodic table. Major impurities were $\mathrm{O}$ (602 ppm at.), $\mathrm{C}$ (189), F (111), and Fe (6). The Si and Ge, both better than 99.99 wt.\% pure, were purchased from commercial vendors. A button weighing approximately $10 \mathrm{~g}$ total was remelted seven times to ensure homogeneity. According to x-ray powder diffraction data the as-prepared sample was a single phase material (within the sensitivity of the technique, which can be estimated as 2-5 vol.\% of an impurity phase) crystallizing in the monoclinic $\mathrm{Gd}_{5} \mathrm{Si}_{2} \mathrm{Ge}_{2}$-type structure.

Magnetic measurements were performed in a commercial (Quantum Design) superconducting quantum interference device (SQUID) magnetometer in applied magnetic fields up to $50 \mathrm{kOe}$ in the temperature range $2-300 \mathrm{~K}$. For zero-fieldcooled (ZFC) measurements of the magnetization, the sample was cooled from the PM state in a zero applied field and the magnetization was measured while warming the sample. The magnetization data in the field-cooled (FC) state were collected while cooling the sample (FCC) in an applied field. Data were also collected while warming the sample in the presence of the field after cooling in the same field (FCW). Several magnetization versus field $(M-H)$ isotherms in fields up to $50 \mathrm{kOe}$ were measured at different temperatures, cooling the sample in a zero magnetic field every time from a true PM state to the temperature of the measurement.

Pressure experiments were carried out using a commercial miniature piston-cylinder-type $\mathrm{CuBe}$ pressure cell Mcell 10 by EasyLab. ${ }^{24}$ The applied pressure was estimated from the superconducting critical temperature using a $\mathrm{Sn}$ manometer. The sample and the Sn manometer were compressed in a Teflon capsule filled with a liquid pressure-transmitting medium (a mixture of mineral oils). Technical details about the pressure cell can be found in Ref. 24. The magnetization was measured under hydrostatic pressures up to $10 \mathrm{kbar}$.

\section{RESULTS AND DISCUSSION}

Figure 1(a) shows the temperature dependence of the real part of the ac magnetic susceptibility $\left(\chi_{a c}^{\prime}\right)$ of $\mathrm{Dy}_{5} \mathrm{Si}_{3} \mathrm{Ge}$ measured at ambient pressure with an ac magnetic field of $H_{a c}=1$ Oe and a frequency of $1 \mathrm{kHz}$. A pronounced cusp at $76 \mathrm{~K}$ for both cooling and warming modes is related to the FM transition according to previous work by Ivtchenko. ${ }^{25}$ The peak at higher temperatures is related to the AFM transition $\left(T_{N} \sim\right.$ $100 \mathrm{~K}$ ) whereas the minor anomaly at $T^{*} \sim 126 \mathrm{~K}$ may be related to the onset of the GP as discussed below. On the other hand, the shoulder observed at temperatures lower than $T_{C}$, around $60 \mathrm{~K}$ on cooling mode indicates the partial structural transformation $O(I) \longleftrightarrow M$ in the FM state already noted by Nirmala et al. ${ }^{22}$ This is supported by the hysteretic behavior usually observed in first-order structural transformations. As expected, the structural transition is slightly decoupled from the FM transition by a temperature span of about $15 \mathrm{~K}$. The observed hysteresis is related to the first-order nature of the near structural transition.

ZFC, FCC, and FCW magnetization data of $\mathrm{Dy}_{5} \mathrm{Si}_{3} \mathrm{Ge}$ obtained in an applied field of 100 Oe at ambient pressure are shown in Fig. 1(b). The AFM transition is observed as a broad hump centered at $T_{N} \sim 100 \mathrm{~K}$, which is preceded by the FM transition at $T_{C} \sim 76 \mathrm{~K}$ during the $\mathrm{ZFC}$ measurements. The FM transition is marked by a significant irreversibility between the ZFC and FC data. The ZFC magnetization decreases below $T_{C}$ with decreasing temperature, whereas both the FCC and FCW magnetizations increase down to $2 \mathrm{~K}$. The difference between the ZFC and FCC magnetization data in ferromagnets is in general related to the coercivity and domain wall pinning. The 


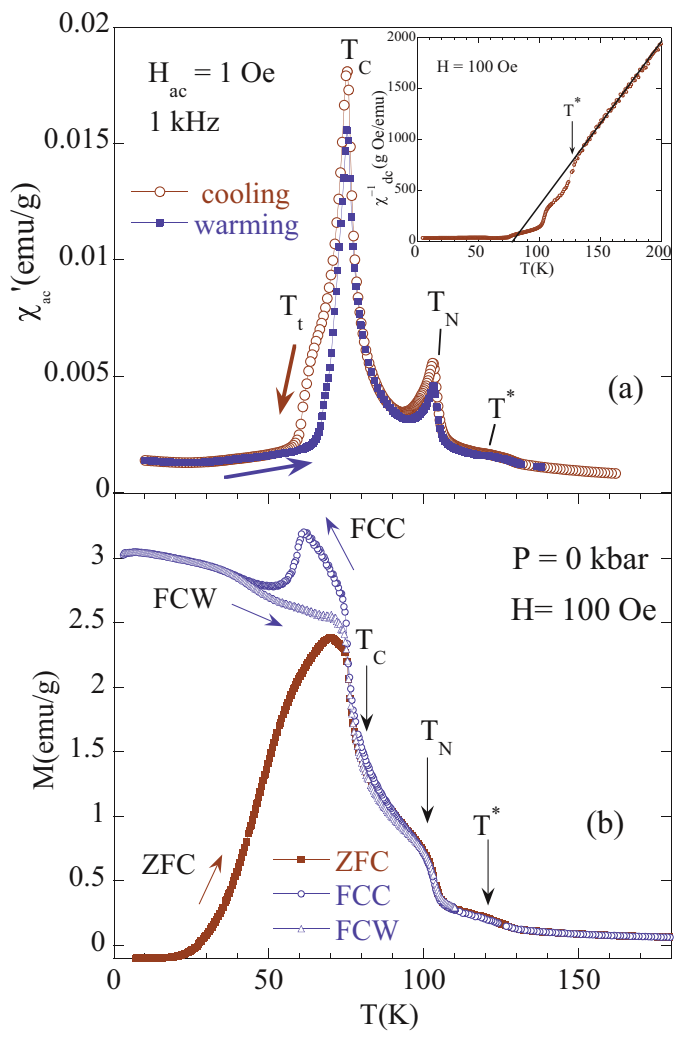

FIG. 1. (Color online) (a) Temperature dependence of the real $\left(\chi_{a c}^{\prime}\right)$ component of the ac susceptibility in an applied ac field of $1 \mathrm{Oe}$ at a frequency of $1 \mathrm{kHz}$. Filled symbols are used for the heating runs and open symbols are used for the cooling curves. (b) ZFC-FC magnetizations (as warming and as cooling) of $\mathrm{Dy}_{5} \mathrm{Si}_{3} \mathrm{Ge}$ as a function of temperature measured at ambient pressure in an applied field of 100 Oe. The inset of Fig. 1(a) shows the Curie-Weiss fit to the inverse magnetic susceptibility in $100 \mathrm{Oe}$, field-cooled state.

large difference observed below $T_{C}$ in the present case reflects sizable pinning effects in this compound. ${ }^{21}$ It is noteworthy that the transition temperatures observed in the sample studied in this work are shifted to higher values than those observed in a sample with the same nominal composition previously investigated by Nirmala et al., ${ }^{21}$ although the sequence and the character of the different magnetic and structural transitions remain unchanged. We attribute these differences to small changes in the composition between both samples. It is worth noting that previous works on 5:4 compounds reveal texture in the polycrystalline samples. ${ }^{26,27}$ This fact together with the strong magnetocrystalline anisotropy of the $\mathrm{Dy}^{3+}$ can explain also the differences found in the magnitude of the low-temperature FC magnetization from previous work by Nirmala et al. $^{21}$

The structural transformation $O(I)+M \longleftrightarrow M$ in the FM regime is detected by a remarkable change in the FCC and FCW magnetization as seen in Fig. 1(b). A steplike anomaly is observed in the magnetization between $\sim 74 \mathrm{~K}$ and $\sim 42 \mathrm{~K}$ on warming, whereas a pronounced maximum centered at $65 \mathrm{~K}$ occurs while cooling. There is a large thermal hysteresis between the warming and the cooling magnetization curves. The width of thermal hysteresis between both curves amounts to about $30 \mathrm{~K}$. This observation agrees with previous

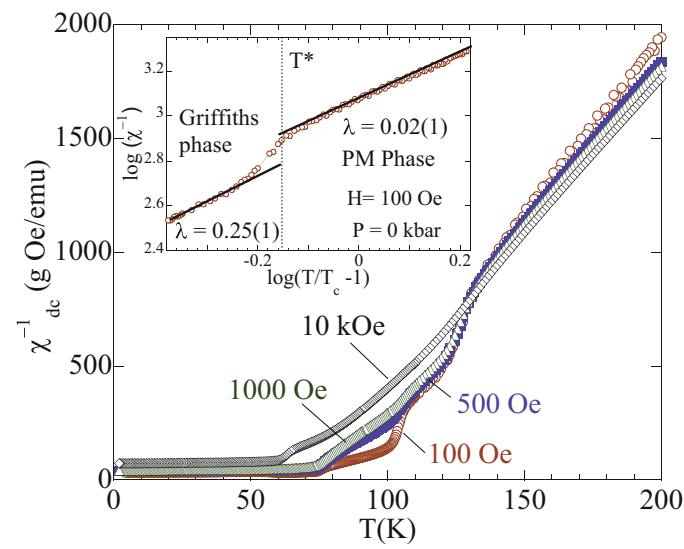

FIG. 2. (Color online) Temperature dependence of reciprocal susceptibility $\chi^{-1}$ of $\mathrm{Dy}_{5} \mathrm{Si}_{3} \mathrm{Ge}$ as a function of magnetic field, measured on heating. The line through the points shows the CurieWeiss fit to the susceptibility. The inset shows fits at $100 \mathrm{Oe}$ in the Griffiths and PM phases, respectively.

work, ${ }^{21}$ which reported a first-order transformation developing in this temperature range. In the present case, however, the crystallographic transition spans a significantly larger temperature range, which suggests an extended first-order transformation due to disorder. ${ }^{28}$ Furthermore, the significant differences between the anomalies in the FCC and FCW magnetizations suggest that not only the structural changes are developing in the FM state but also different FM ordered structures can be present in the $M$ and $O(\mathrm{I})$ phases, as it was shown in an $\mathrm{Er}_{5} \mathrm{Si}_{4}$ compound by neutron scattering measurements. ${ }^{15}$

Inset of Fig. 1(a) shows the low-field reciprocal susceptibility $\left(\chi^{-1}=H / M\right)$ at 100 Oe. On cooling, $\chi^{-1}$ exhibits a linear thermal dependence (as expected from the Curie-Weiss law) that however disappears at $T^{*}$ above $T_{N}$. Above $T^{*}$, the susceptibility obeys the Curie-Weiss law $\left[M / H=C /\left(T-\theta_{P}\right)\right]$ and yields a paramagnetic Weiss temperature $\left(\theta_{P}\right)$ of $75 \mathrm{~K}$ and an effective moment $\mu_{\mathrm{eff}}=$ $11.8 \mu_{B} / \mathrm{Dy}^{3+}$, respectively. The experimentally determined value is slightly larger than the theoretical value for the $\mu_{B} / \mathrm{Dy}^{3+}$ free ion $\left(g[J(J+1)]^{1 / 2}=10.65 \mu_{B}\right)$. The positive value of $\theta_{P}$ indicates that FM interactions are dominant in this compound in agreement with previous studies. ${ }^{21}$ Between $T^{*}$ and $T_{N}$ the inverse susceptibility shows a negative deviation from the Curie-Weiss behavior, which is often considered as a signature of a GP. In fact, on further increasing the magnetic field, the anomaly displayed in $\chi^{-1}$ is suppressed, becoming nearly indistinguishable from the high-temperature range values at $H>10 \mathrm{kOe}$ (see Fig. 2). Furthermore, a magnetic susceptibility exponent $\lambda$ lower than unity, i.e., $\chi^{-1}(T)=\left(T / T_{C}-1\right)^{1-\lambda}$, where $0 \leqslant \lambda<1$, is obtained in this temperature range. We have fitted the logarithmic representation of $\chi^{-1}$ (see inset in Fig. 2) obtaining clearly different values for the exponent depending on whether we refine it in the anomalous region of $\chi^{-1}\left[T_{N}<T<T^{*}, \lambda_{G}=0.25(1)\right.$ at $100 \mathrm{Oe}]$ or in the conventional PM phase $\left[T>150 \mathrm{~K}, \lambda_{P M}=\right.$ $0.02(1)$ at $100 \mathrm{Oe}]$. This behavior, as previously seen in similar compounds such as $\mathrm{Tb}_{5} \mathrm{Si}_{2} \mathrm{Ge}_{2}$ and $\mathrm{Gd}_{5} \mathrm{Ge}_{4},{ }^{7,29}$ is typical of short-range FM clusters in a PM matrix and is the hallmark of 
Griffiths singularities. ${ }^{30,31}$ This result is in agreement with the presented $(x, T)$ phase diagram of the system $\operatorname{Dy}_{5}\left(\mathrm{Si}_{x} \mathrm{Ge}_{1-x}\right)_{4}$, which predicts $T^{*}$ at $\sim 125 \mathrm{~K}$ for $\mathrm{Dy}_{5} \mathrm{Si}_{3} \mathrm{Ge}(x=0.75) .{ }^{8} \mathrm{At}$ higher fields, the values of the exponent still remain far from zero value (not shown), e.g., $\lambda_{G}(1000 \mathrm{Oe})=0.12(2)$, so the $\mathrm{GP}$ exists at fields higher than $1 \mathrm{kOe}$. In contrast, $\lambda_{P M}$ remains quite small $[\sim 0.04(1)]$ indicating that the GP does not extend to temperatures higher than $\sim 150 \mathrm{~K}$.

Temperature-dependent dc magnetization measurements as a function of hydrostatic pressure at low field $(H=100 \mathrm{Oe})$ are shown in Fig. 3. The effect of the applied pressure indicates that low pressure $(P<4$ kbar) induces a notable change in the structurally phase-separated magnetic state of $\mathrm{Dy}_{5} \mathrm{Si}_{3} \mathrm{Ge}$ below $T_{C}$. The pronounced maximum at $63 \mathrm{~K}$ in the $\mathrm{FCC}$ magnetization at ambient pressure, which coincides with the structural transition, shifts towards higher temperatures and rapidly decreases upon increasing pressure. At $4 \mathrm{kbar}$, the structural transition appears as a steplike anomaly at $73 \mathrm{~K}$. Such a displacement is equivalent to a rate of $d T_{t} / d P \sim$ $2.5 \mathrm{~K} / \mathrm{kbar}$. The width of the thermal hysteresis significantly decreases upon application of relatively low pressure: from $\Delta T=30 \mathrm{~K}$ at ambient pressure down to $\Delta T \sim 8 \mathrm{~K}$ at 4 kbar. For pressures above 4 kbar it remains unaffected. This observation indicates that the transition retains its firstorder character within the pressure range studied although significant changes are induced at low pressures.

Figure 4 shows the dependence of the different phase transitions $T_{C}, T_{N}$, and $T_{t}$ with pressure obtained from the data shown in Fig. 3. The transition temperatures $\left(T_{C}\right.$ and $\left.T_{N}\right)$ have been estimated from the maximum of the derivative $|d M / d T|$ whereas the structural transition $T_{t}$ has been estimated from the maximum in the FCC magnetization curve. The AFM transition in the $M$ phase shifts linearly with pressure to higher temperatures at a slow rate of $d T_{N} / d P=0.34 \mathrm{~K} / \mathrm{kbar}$, which is expected for a pure magnetic anomaly. However, the FM transition in the $M$ phase shifts linearly with pressure at a slow rate of $d T_{C} / d P=0.2 \mathrm{~K} / \mathrm{kbar}$ for pressures below $4 \mathrm{kbar}$, showing a strong dependence for pressures above $4 \mathrm{kbar}\left(d T_{C} / d P=4.7 \mathrm{~K} / \mathrm{kbar}\right)$. As it is clearly seen in Fig. 3(c), at 4 kbar the structural transition (maximum in FCC magnetization) is in the vicinity of the FM transition $T_{C}(\sim 78 \mathrm{~K})$. The proximity between the low-temperature crystallographic $M-O(\mathrm{I})$ and FM transition causes both to collapse at higher pressures (above $4 \mathrm{kbar}$ ) giving rise to the observed enhancement of the pressure rate of $T_{C}$. Increasing the pressure up to $4 \mathrm{kbar}$ induces a significant increase of the low-temperature magnetization [Figs. 3(a) and 3(c)], which is nearly $30 \%$ the ambient pressure value. These results indicate that low pressure $(P<4 \mathrm{kbar})$ induces significant changes in the magnetic state of $\mathrm{Dy}_{5} \mathrm{Si}_{3} \mathrm{Ge}$.

The effect of hydrostatic pressure on the onset of the GP in the PM regime, which is detected by the anomaly displayed in the inverse of susceptibility at $T^{*}$ at 100 Oe at ambient pressure, can be analyzed. The temperature dependence of the inverse susceptibility at 100 Oe under selected pressures is depicted in Fig. 5. The negative deviation from Curie-Weiss is observed for all the measured pressures. We have fitted the logarithmic representation of $\chi^{-1}$ obtaining values of the exponent $\lambda$ far from zero in the GP state $T_{C}<T<T^{*}$ and $\lambda_{P M} \sim 0$ for $T>T^{*}$. Those fits are shown in Fig. 6

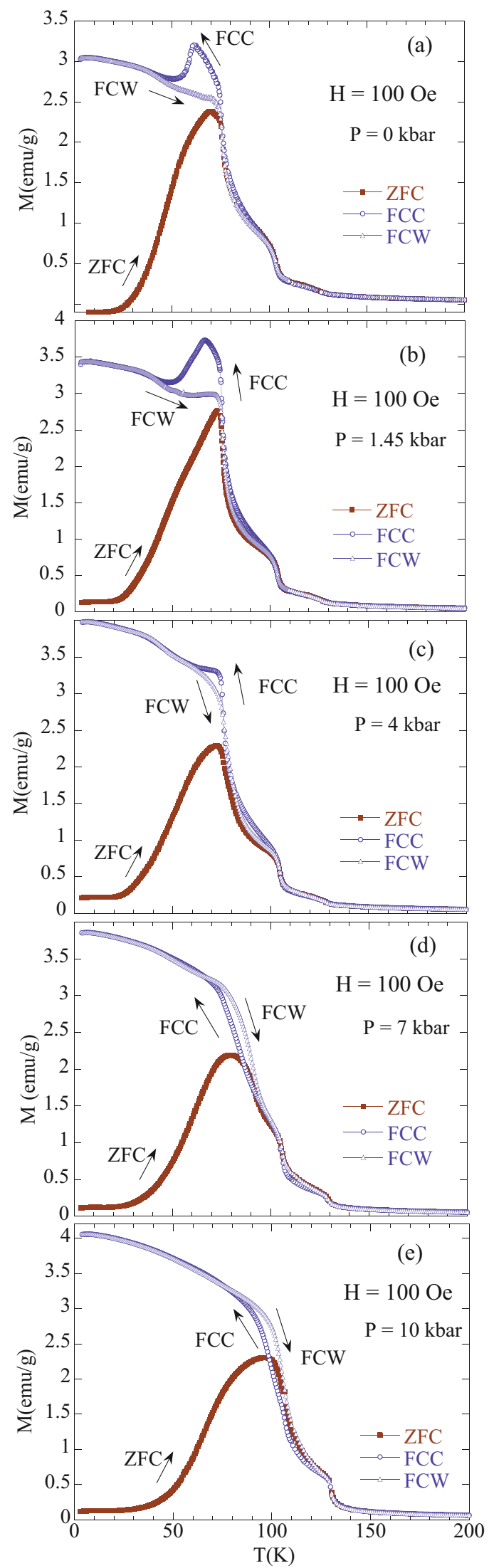

FIG. 3. (Color online) FCC, FCW, and ZFC magnetizations (as warming and as cooling) of $\mathrm{Dy}_{5} \mathrm{Si}_{3} \mathrm{Ge}$ as a function of temperature and hydrostatic pressure measured in an applied field of 100 Oe at (a) 0 kbar, (b) $1.45 \mathrm{kbar}$, (c) $4 \mathrm{kbar}$, (d) $7 \mathrm{kbar}$, and (e) $10 \mathrm{kbar}$. 


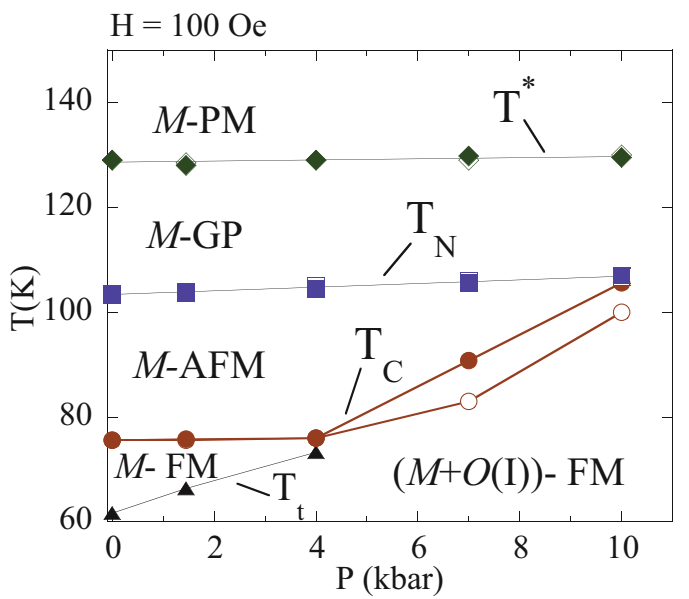

FIG. 4. (Color online) $T-P$ crystallographic-magnetic phase diagram for $\mathrm{Dy}_{5} \mathrm{Si}_{3} \mathrm{Ge}$ as obtained from magnetization measurements at 100 Oe. Diamonds represent the onset of the GP phase $\left(T^{*}\right)$, squares are used for the AFM transition $\left(T_{N}\right)$, circles represent the onset of the FM order $\left(T_{C}\right)$, whereas triangles are used for the structural transformation $\left(T_{t}\right)$. Open symbols are used in the cooling curves (FCC) and solid symbols in the heating runs (FCW). Thick solid lines depict the magnetic and/or crystallographic phase boundaries.

for selected pressures as representative of this study. It is worth noting that for each analysis the shift of $T_{C, N}$ with pressure has been taken into account. These measurements indicate that GP remains at higher pressures up to $10 \mathrm{kbar}$ $\left[\lambda_{P M} \sim 0\right.$ and $\left.\lambda_{G} \sim 0.18(2)\right]$. A slight variation of the exponent $\lambda_{G}$ is observed in the investigated pressure range, and $T^{*}$ shifts to higher temperatures at a very slow rate of $d T^{*} / d P \sim 0.16 \mathrm{~K} / \mathrm{kbar}$ (see Fig. 4). Such a pressure dependence of $T^{*}$ is in good agreement with the reported behavior on $R_{5}\left(\mathrm{Si}_{x} \mathrm{Ge}_{1-x}\right)_{4}$ series $(R=\mathrm{Gd}$, Tb, Dy, and $\mathrm{Ho}) .{ }^{8}$ According to that study, $T^{*}$ shifts to higher temperatures as $\mathrm{Si}$ content increases (i.e., as the cell volume decreases) in all the studied magnetic and structural phase diagrams. It is worth noting that a direct comparison between the hydrostatic and chemical pressure effects is not suitable since the change

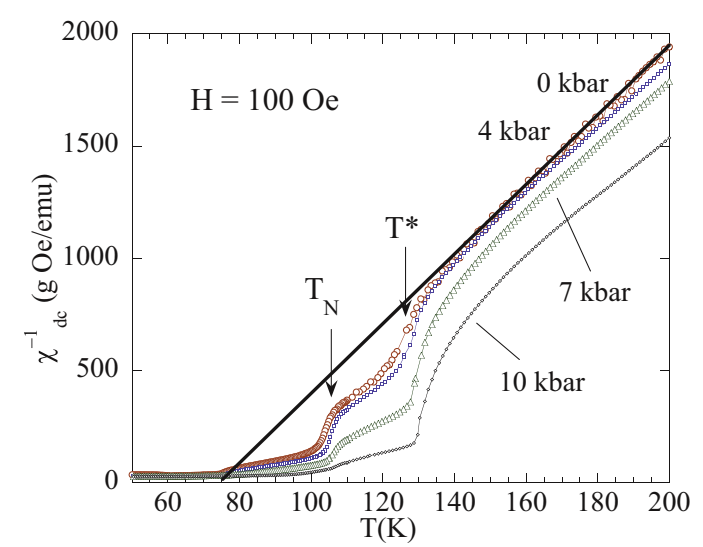

FIG. 5. (Color online) Temperature dependence of $\chi^{-1}$ of $\mathrm{Dy}_{5} \mathrm{Si}_{3} \mathrm{Ge}$ at $100 \mathrm{Oe}$ at selected hydrostatic pressures. Thick solid line depicts the Curie-Weiss fit to the inverse magnetic susceptibility in $100 \mathrm{Oe}$, at $0 \mathrm{kbar}$.
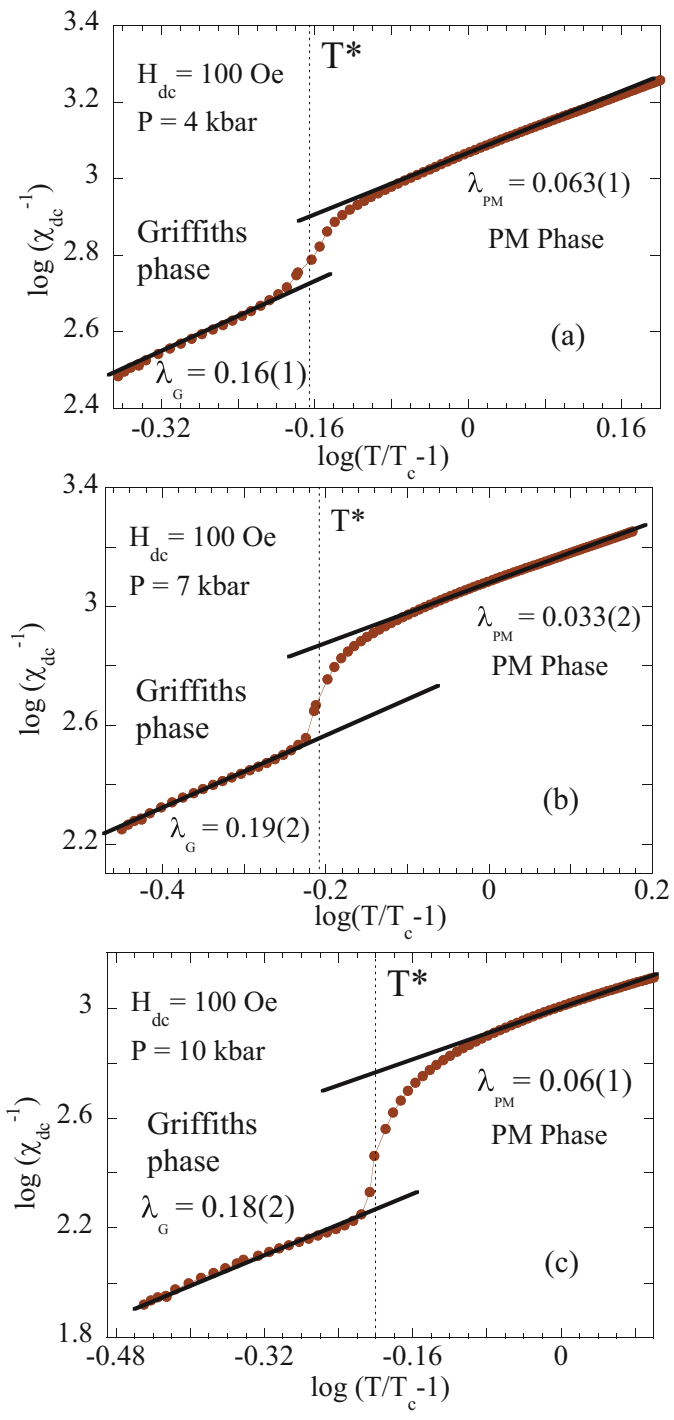

FIG. 6. (Color online) Linear fits of $\chi^{-1}$ vs $\left(T / T_{C}-1\right)$ in double logarithmic scale near $T^{*}$. The different curves are labeled with the corresponding pressure values.

of the Si content not only modifies the cell volume but also introduces changes in the electronic structure of the compound.

Figure 7 illustrates the dc magnetization data collected in an applied field of $1 \mathrm{kOe}$. The ambient pressure measurement resembles the low-field curve shown in Fig. 1 with an obviously higher magnetic signal. The AFM transition is quenched by the field whereas the anomaly displayed in $\chi^{-1}$ at $T^{*}$ shown in Fig. 2 still remains. The structural transition appears as a steplike anomaly between $\sim 70 \mathrm{~K}$ and $\sim 40 \mathrm{~K}$ on both warming and cooling curves with thermal hysteresis between the two curves. An applied field of 1 kOe has strongly reduced the maximum in the FCC magnetization seen at 100 Oe. The application of pressure induces two main processes: first is the shift of the FM transition towards higher temperatures. The second is the appearance of large thermal hysteresis of $\sim 6 \mathrm{~K}$ between the FCC and FCW curves observed around $T_{C}$ for pressures above $4 \mathrm{kbar}$. During the pressure application process the maximum of the derivative $|d M / d T|$ 

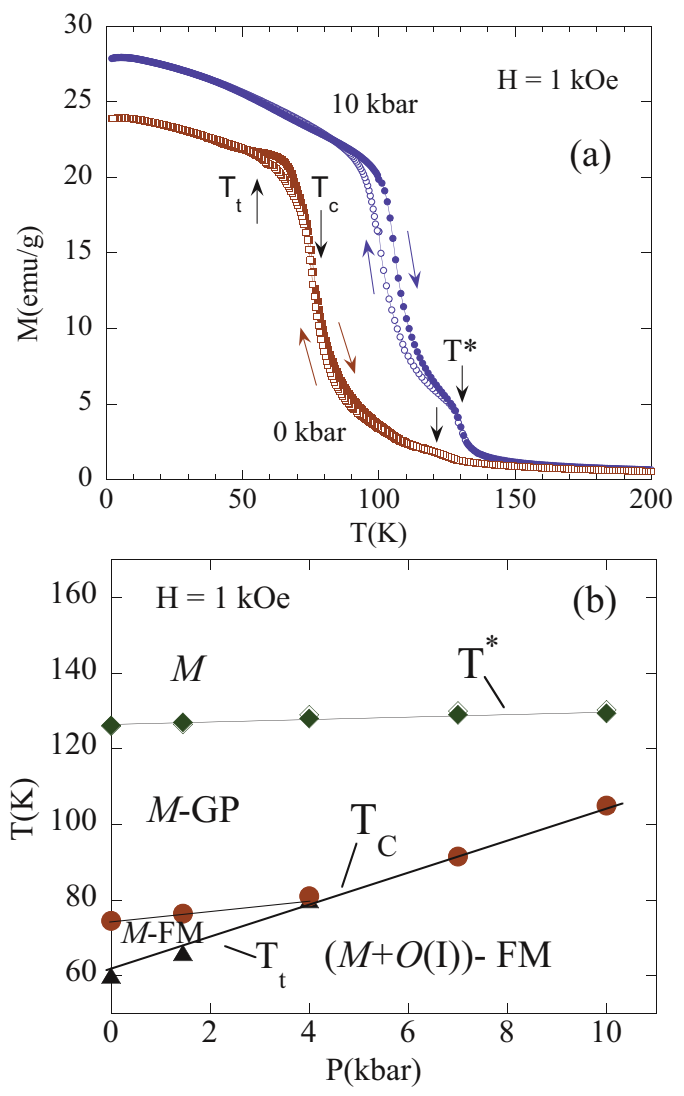

FIG. 7. (Color online) (a) $\mathrm{FC}$ magnetizations of $\mathrm{Dy}_{5} \mathrm{Si}_{3} \mathrm{Ge}$ measured in $1 \mathrm{kOe}$ magnetic field as a function of temperature at ambient pressure and $10 \mathrm{kbar}$ during heating (FCW) and cooling (FCC). Open symbols are used in the cooling curves and solid symbols in the heating runs. The different transitions are marked by arrows (see text for details). (b) $T$ - $P$ magnetic phase diagram for $\mathrm{Dy}_{5} \mathrm{Si}_{3} \mathrm{Ge}$ as obtained from magnetization measurements at $1 \mathrm{kOe}$. Diamonds represent the onset of the GP phase $\left(T^{*}\right)$, circles represent the onset of the FM order $\left(T_{C}\right)$ whereas triangles are used for the structural transformation $\left(T_{t}\right)$. Open symbols are used in the cooling curves (FCC) and solid symbols in the heating runs (FCW). Thick solid lines depict the magnetic and/or crystallographic phase boundaries.

has shifted linearly with pressure from its initial temperature of $T_{C}=77 \mathrm{~K}$ up to $T_{C}=80 \mathrm{~K}$ at $4 \mathrm{kbar}$. Such a displacement corresponds to a slow rate of $d T_{C} / d P \sim 0.32 \mathrm{~K} / \mathrm{kbar}$, similar to the obtained rate at low magnetic fields in the same pressure range [see Fig. 7 (b)]. Above 4 kbar, however, the linear shift of the transition with pressure corresponds to a rate of $d T_{C} / d P \sim 4.0 \mathrm{~K} / \mathrm{kbar}$. The large thermal hysteresis around $T_{C}$ points to the first-order nature of the transformation at $T_{C}$. These results suggest that a coupled magnetic-crystallographic transformation takes place at pressures above $4 \mathrm{kbar}$, and thus the increase of the transition temperature with pressure is one order of magnitude higher. It is noteworthy that the low-temperature magnetization increases with rising pressure a $17 \%$ the ambient pressure value. On the other hand, GP persists at pressures up to $10 \mathrm{kbar}\left[\lambda_{P M} \sim 0\right.$ and $\lambda_{G} \sim$ $0.14(2)]$. The value of $T^{*}$, associated with the onset of short-range FM correlations, increases linearly with pressure at a pressure rate $d T^{*} / d P=0.32 \mathrm{~K} / \mathrm{kbar}$, which is higher
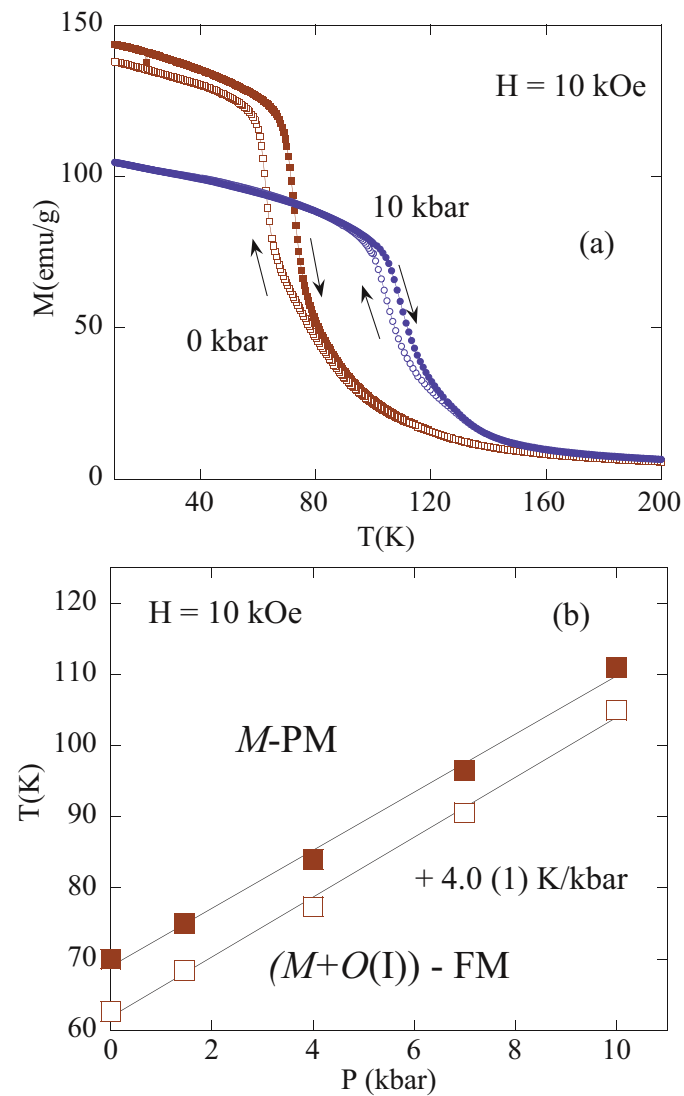

FIG. 8. (Color online) (a) FC magnetizations of $\mathrm{Dy}_{5} \mathrm{Si}_{3} \mathrm{Ge}$ measured in $10 \mathrm{kOe}$ magnetic field as a function of temperature at ambient pressure and 10 kbar during heating (FCW) and cooling (FCC). Open symbols are used in the cooling curves and solid symbols in the heating runs. (b) Pressure-temperature magnetic phase diagram for $\mathrm{Dy}_{5} \mathrm{Si}_{3} \mathrm{Ge}$ as obtained from magnetization measurements at $10 \mathrm{kOe}$. Open symbols are used in the cooling curves (FCC) and solid symbols in the heating runs (FCW).

than the observed rate at $H=100$ Oe. This fact indicates a strong interplay between the applied magnetic field and the hydrostatic pressure on $T^{*}$. As far as we know, theoretical studies about the effect of hydrostatic pressure on the GP are still lacking. We believe that our work gives interesting experimental results in order to understand the dependence of the GP on $P$ and $H$.

The interesting behavior of $\mathrm{Dy}_{5} \mathrm{Si}_{3} \mathrm{Ge}$ is further clarified by the high-field dc magnetization measurements ( $H=10 \mathrm{kOe})$. Temperature dependencies of the high-field magnetization at different pressure values are displayed in Fig. 8(a). Only zero and maximum pressures are included for the sake of clarity. At ambient pressure, an applied field of $10 \mathrm{kOe}$ quenches the AFM transition and the anomaly displayed by $\chi^{-1}$ at $T^{*}$ and low field is suppressed (see Fig. 2). A large thermal hysteresis of $\Delta T \sim 8 \mathrm{~K}$ between the FCC and FCW magnetization at zero pressure around $T_{C}$ is observed. The corresponding $T-P$ phase diagram is shown in Fig. 8(b) where the transition temperature values have been taken at the maximum derivative of the magnetization. It is easy to see that the magnetic transformation moves linearly with pressure towards higher temperature values at a rate of $d T_{C} / d P=4.1 \mathrm{~K} / \mathrm{kbar}$ on 
cooling and $4.3 \mathrm{~K} / \mathrm{kbar}$ on warming. Moreover, the transition retains its first-order character within the pressure range studied as can be deduced from the hysteretic behavior and from the little variation in the sharpness of the anomaly. The width of the hysteresis however, decreases with pressure down to $\Delta T \sim 5 \mathrm{~K}$. These observations suggest that the high-temperature second-order FM transition is coupled with the low-temperature first-order structural phase change into a first-order magnetic-crystallographic transformation, this coupling being induced by a high magnetic field $(10 \mathrm{kOe})$. Remarkable changes are being induced by pressure in the high-field magnetic state of $\mathrm{Dy}_{5} \mathrm{Si}_{3} \mathrm{Ge}$ as can be inferred from the significant decrease of the low-temperature magnetization [Fig. 8(a)] upon increasing pressure. The decrease of the low-temperature magnetization observed at $10 \mathrm{kOe}$ is opposite to the behavior observed at lower magnetic field [see Fig. 7(a)]. This fact can be due to the different ratio of volume fractions of the $M$ and $O(\mathrm{I})$ phases as a function of $P$ and $H$. The $O(\mathrm{I})$ phase is favored by both the hydrostatic pressure and the applied magnetic field. The effect of $H$ can be clearly seen in the phase diagrams shown in Figs. 7(b) and 8(b): At ambient pressure, the temperature region where only the $M$ phase is present is strongly reduced by the applied magnetic field and disappears at $10 \mathrm{kOe}$. The enhancement of the $O(\mathrm{I})$ phase content with applied magnetic field has been also observed in the $\mathrm{Er}_{5} \mathrm{Si}_{4}$ system with neutron scattering experiments. ${ }^{32}$ At low applied magnetic field $(H<10 \mathrm{kOe})$ and low temperatures, the main effect of pressure is to transform the remanent $M$ phase content into the $O(\mathrm{I})$ phase with high magnetic moment. As a consequence, an increase of the low-temperature magnetization is observed. At applied magnetic fields higher than $10 \mathrm{kOe}$, the $O(\mathrm{I})$ phase content is close to $100 \%$ even at ambient pressure. In this case, the applied pressure modifies the magnetic behavior of the $O(\mathrm{I})$ phase. The reduction of the low-temperature magnetization seen in Fig. 8 can be explained either as assuming that the hydrostatic pressure induces a more canted ferromagnetic structure in the $O(\mathrm{I})$ phase or considering that $P$ modifies the magnetocrystalline anisotropy present in this compound. The latter would change the magnetic isotherms giving rise to a reduction of the magnetization as pressure increases. This behavior has already been observed in an $\mathrm{Er}_{5} \mathrm{Si}_{4}$ single crystal under pressure. $^{16}$

Magnetization has been measured as a function of field at various temperatures in fields up to $50 \mathrm{kOe}$ at selected hydrostatic pressures ranging from 0 to $\sim 10 \mathrm{kbar}$ (values at low temperature). The dependence of the magnetization isotherms at $5 \mathrm{~K}$ on the hydrostatic pressure is illustrated in Fig. 9. A change in the magnetic state of $\mathrm{Dy}_{5} \mathrm{Si}_{3} \mathrm{Ge}$ with pressure is seen when comparing the ambient pressure isotherm with the applied-pressure measurements. At ambient pressure, a metamagnetic process takes place at $\sim 7 \mathrm{kOe}$ followed by a significant increase in magnetization, which is then followed by a hysteretic behavior. Magnetization smoothly increases in the high magnetic field range without reaching the saturation. At $50 \mathrm{kOe}$ the magnetization reaches $\sim 7 \mu_{B}$ /Dy, which is significantly lower than the theoretical saturation magnetization moment $\left(g J=10 \mu_{B}\right)$ for the $\mathrm{Dy}^{3+}$ ion. On the other hand, the magnetic hysteresis is rather large and substantial coercivity and remanent magnetization values

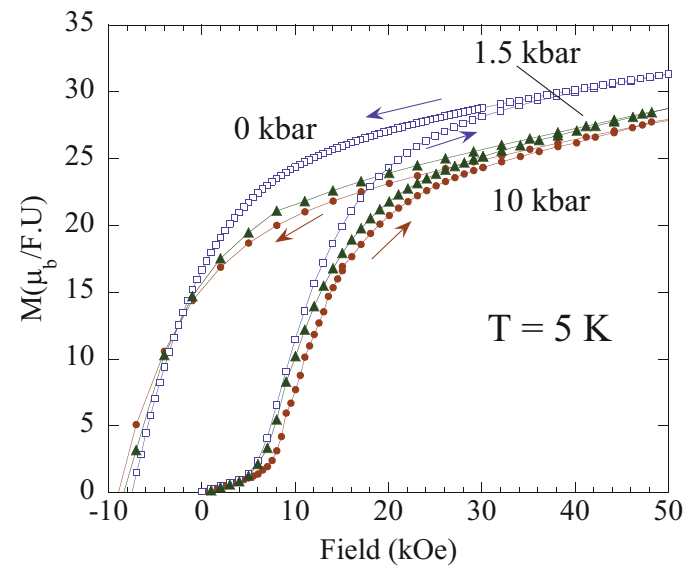

FIG. 9. (Color online) Magnetization isotherms of $\mathrm{Dy}_{5} \mathrm{Si}_{3} \mathrm{Ge}$ measured at $5 \mathrm{~K}$ at selected pressures.

( $\sim 7 \mathrm{kOe}$ and $\sim 100 \mathrm{emu} / \mathrm{g}$, respectively) are found. It may be mentioned that, in general, the magnetic $R_{5} T_{4}$ materials with a large anisotropy show considerable hysteresis and possess large value of coercivity and remanence. ${ }^{21}$ It is worth noting that some discrepancies in the shape of $M(H)$ curves compared to previous results are related to texture that is always present in the as-solidified alloys. ${ }^{21}$ In our sample the magnetic field was likely applied along a direction close to the hard magnetization direction and the sample is not saturated at $50 \mathrm{kOe}$. This is clearly observed both in the slope of the magnetization at high fields and in the low value of the magnetic moment compared to the expected for the $\mathrm{Dy}^{3+}$ ion and the obtained by Nirmala et $a l .{ }^{21}$ Moreover, a canted ferromagnetic structure as is commonly observed in $R_{5} T_{4}$ compounds can also contribute to the low magnetic moment obtained.

A pressure of $1.5 \mathrm{kbar}$ shifts the metamagnetic transition to higher magnetic fields $\sim 8.5 \mathrm{kOe}(\sim 20 \%$ higher than the ambient pressure value) whereas the magnetization reaches $\sim 5.6 \mu_{B}$ /Dy at $50 \mathrm{kOe}(\sim 15 \%$ lower than the ambient pressure value). On the other hand, coercivity and remanent magnetization values are $\sim 8.5 \mathrm{kOe}$ and $\sim 90 \mathrm{emu} / \mathrm{g}$, respectively. It is worth noting that this behavior remains essentially unchanged at higher pressures, so the changes induced by pressure at $5 \mathrm{~K}$ are already accomplished at $1.5 \mathrm{kbar}$.

Figure 10(a) displays the measurements carried out in the vicinity of $T_{t}$ at $50 \mathrm{~K}$, whereas Fig. 10(b) shows the isotherms in the vicinity of $T_{C}$ at $75 \mathrm{~K}$. At $T=50 \mathrm{~K}$ (close to the structural transition and $20 \mathrm{~K}$ lower than $T_{C}$ ), the ambient pressure magnetization shows a fast increase at low fields (up to $\sim 8 \mathrm{kOe}$ ) associated with the displacement of the domain walls, a typical behavior for ferromagnets. After that, the magnetization smoothly increases with the magnetic field. At higher fields (above $30 \mathrm{kOe}$ ) it shows little tendency toward saturation, reaching $\sim 6 \mu_{B}$ /Dy at the maximum field $(50 \mathrm{kOe})$. Figure 10(a) evidences a substantial change in the magnitude of the magnetization at low pressure, whereas the shape remains unaffected when pressure increases up to $1.5 \mathrm{kbar}$. For higher pressures the magnetization remains unaffected. At $50 \mathrm{kOe}$ the magnetization value changes from $\sim 30 \mu_{B} /$ F.U at ambient pressure to $\sim 26 \mu_{B} / \mathrm{F}$.U at the maximum pressure (10 kbar), which is equivalent to a significant decrease of $\sim 15 \%$. 

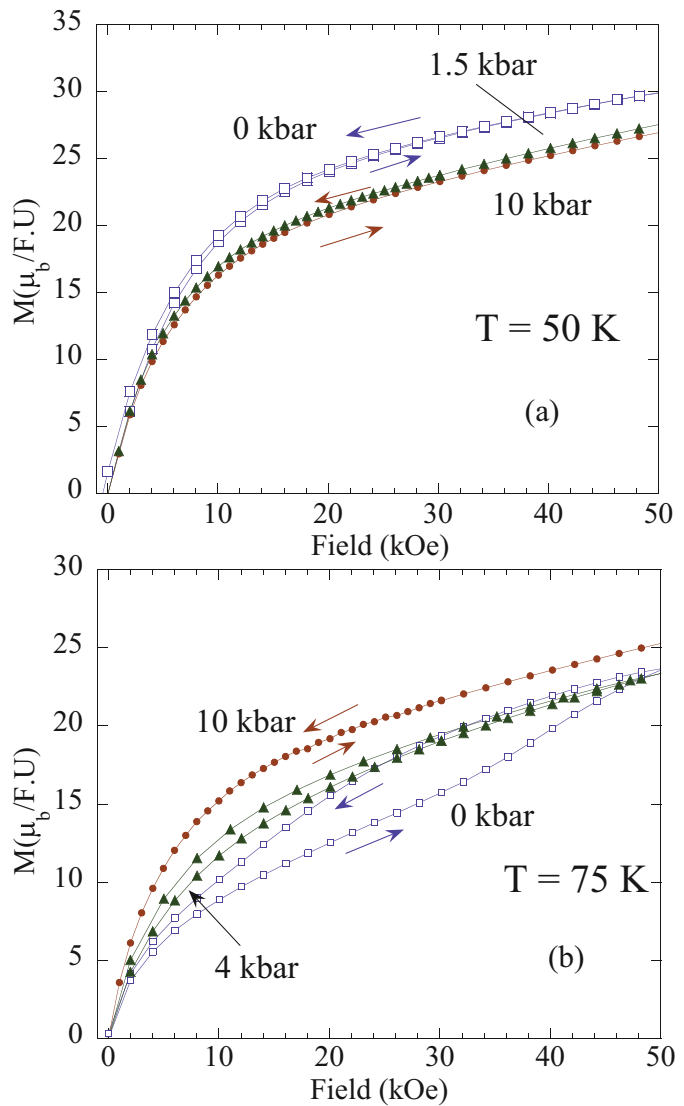

FIG. 10. (Color online) Magnetization isotherms of $\mathrm{Dy}_{5} \mathrm{Si}_{3} \mathrm{Ge}$ measured at selected pressures and temperatures in the vicinity of structural transition and magnetic ordering: (a) at $50 \mathrm{~K}$ and (b) at $75 \mathrm{~K}$.

At $T=75 \mathrm{~K}$ (well above $T_{t}$, close to $T_{C}$ and below $T_{N}$ ) the ambient pressure isotherm shows a moderate increase at low fields (up to $\sim 5 \mathrm{kOe}$ ) associated with the displacement of the domain walls [Fig. 10(a)]. With a further increase of the field the magnetization increases rather slowly and shows a broad metamagneticlike transition in the field range of 25-30 kOe, which is followed by a hysteretic behavior associated to the structural transition. ${ }^{18}$ A significant change in the shape of the magnetization is observed when pressure increases up to $4 \mathrm{kbar}$. The application of $4 \mathrm{kbar}$ suppresses the metamagneticlike transition and the increase of the magnetization at low fields is more rapid. The magnetization value at the maximum field however, remains unaffected. Upon further increasing of the pressure, a significant increase of the magnetization is observed (almost $9 \%$ higher than the ambientpressure value at the maximum field). This enhancement of the magnetization is more pronounced in the magnetic fields below $10 \mathrm{kOe}$, where the high-pressure isotherm exhibits a more rapid tendency toward saturation.

The behavior observed in the isotherms is very complex and difficult to explain considering the high number of parameters present in these measurements. As the magnetic field and hydrostatic pressure are applied we are changing the magnetization in each crystallographic phase $[M$ and $O(\mathrm{I})]$ and simultaneously transforming the $M$ phase into the $O(\mathrm{I})$ phase favored by both $H$ and $P$. Therefore, the magnetization isotherms are a result of the interplay between the different magnetocrystalline anisotropies of the $M$ and $O(\mathrm{I})$ phases and the transformation of the $M$ phase into the $O(\mathrm{I})$ phase. From our magnetization measurements it is clear that at $5 \mathrm{~K}$ and $1.5 \mathrm{kbar}$ the $M$ phase has been completely transformed into the $O(\mathrm{I})$ phase as no changes are present in the magnetization isotherms above that pressure. The reduction in the magnetization observed can be attributed to a higher magnetocrystalline anisotropy of the $O(\mathrm{I})$ phase. For higher temperatures, higher pressures are needed to transform the $M$ phase into the $O(\mathrm{I})$ and close to the Curie temperature the maximum applied pressure is not high enough to completely transform the $M$ into the $O(\mathrm{I})$ phase, and a more complex behavior with magnetic field is observed. For a complete understanding of the effect of the hydrostatic pressure on the magnetocrystalline anisotropy and the phase transformation, measurements of a single crystalline sample are needed.

\section{CONCLUSIONS}

An extensive study of the effects of the hydrostatic pressure and magnetic field on the structural and magnetic phases of the $\mathrm{Dy}_{5} \mathrm{Si}_{3} \mathrm{Ge}$ compound has been performed. From our measurements the magnetic and structural phase diagrams as a function of the magnetic field and the hydrostatic pressure have been constructed. The experimental measurements demonstrate that the $O(\mathrm{I})$ ferromagnetic phase is favoured by applying $H$ and $P$, while the $M$-AFM and $M$-FM regions disappear. A magnetic field of $10 \mathrm{kOe}$ induces the coupling of the second-order FM transition with the low-temperature first-order structural phase change. At lower magnetic fields, such a coupling is induced by hydrostatic pressure ( $\sim 4$ kbar).

The thermal dependence of the magnetization at different $H$ and $P$ has been explained as coexistence of both $M$ and $O$ (I) phases. Moreover, a complete study of the dependence of the Griffiths-like phase with hydrostatic pressure has been carried out. The Griffiths-like phase persists at pressures up to $10 \mathrm{kbar}$. The value of $T^{*}$, associated with the onset of short-range FM correlations, increases linearly with pressure at a rate of $d T^{*} / d P \sim 0.16 \mathrm{~K} / \mathrm{kbar}$, in agreement with the reported behavior on $R_{5}\left(\mathrm{Si}_{x} \mathrm{Ge}_{1-x}\right)_{4}$ series $(R=\mathrm{Gd}, \mathrm{Tb}$, Dy, and Ho). This experimental evidence may stimulate the interest of theoreticians in the development of theoretical models to explain the dependence of the Griffiths phases as a function of different external parameters, such as high magnetic fields and hydrostatic pressure, in order to get a deeper insight on the interplay between external stimuli and the disorder-induced exotic magnetic behavior of Griffiths phases.

\section{ACKNOWLEDGMENTS}

Work at the University of Zaragoza is supported by the Spanish Ministry of Science (MAT2011-27553-C02, MAT2011-27573-C04) and Spanish DGA (Grant No. E26). Work at the Ames Laboratory is supported by the Office of Basic Energy Sciences, Materials Sciences and Engineering Division of the Office of Science of the US Department of Energy. Ames Laboratory is operated by Iowa State University of Science and Technology for the US Department of Energy under Contract No. DE-AC02-07CH11358. 
${ }^{1}$ V. K. Pecharsky and K. A. Gschneidner, Jr., Phys. Rev. Lett. 78, 4494 (1997).

${ }^{2}$ V. K. Pecharsky and K. A. Gschneidner, Jr., Appl. Phys. Lett. 70, 3299 (1997).

${ }^{3}$ L. Morellón, J. Stankiewicz, B. García-Landa, P. A. Algarabel, and M. R. Ibarra, Appl. Phys. Lett. 73, 3462 (1998).

${ }^{4}$ L. Morellon, P. A. Algarabel, M. R. Ibarra, J. Blasco, B. GarcíaLanda, Z. Arnold, and F. Albertini, Phys. Rev. B. 58, R14721 (1998).

${ }^{5}$ V. K. Pecharsky and K. A. Gschneidner Jr., Pure Appl. Chem. 79, 1383 (2007).

${ }^{6}$ W. Choe, V. K. Pecharsky, A. O. Pecharsky, K. A. Gschneidner, Jr., V. G. Young, and G. J. Miller, Phys. Rev. Lett. 84, 4617 (2000).

${ }^{7}$ C. Magén, P. A. Algarabel, L. Morellón, J. P. Araújo, C. Ritter, M. R. Ibarra, A. M. Pereira, and J. B. Sousa, Phys. Rev. Lett. 96, 167201 (2006).

${ }^{8}$ A. M. Pereira, L. Morellón, C. Magén, J. Ventura, P. A. Algarabel, M. R. Ibarra, J. B. Sousa, and J. P. Araújo, Phys. Rev. B 82, 172406 (2010).

${ }^{9}$ C. Magén, L. Morellón, P. A. Algarabel, M. R. Ibarra, Z. Arnold, and C. Ritter, Advances in Solid State Physics (Springer, Berlin, 2007), Vol. 46, pp. 241-253.

${ }^{10}$ V. K. Pecharsky, A. O. Pecharsky, Y. Mozharivskyj, K. A. Gschneidner, Jr., and G. J. Miller, Phys. Rev. Lett. 91, 207205 (2003).

${ }^{11}$ C. Ritter, C. Magén, L. Morellón, P. A. Algarabel, M. R. Ibarra, V. K. Pecharsky, A. O. Tsokol, and K. A. Gschneidner, Jr., J. Phys.: Condens. Matter 18, 3937 (2006).

${ }^{12}$ A. M. Pereira, J. P. Araújo, M. E. Braga, R. P. Pinto, J. Ventura, F. C. Correia, J. M. Teixeira, J. B. Sousa, C. Magén, P. A. Algarabel, L. Morellón, and M. R. Ibarra, J. Alloys Compd. 423, 66 (2006).

${ }^{13}$ F. Holtzberg, R. J. Gambino, and T. R. McGuire, J. Phys. Chem. Solids 28, 2283 (1967).

${ }^{14}$ Y. Mozharivskyj, A. O. Pecharsky, V. K. Pecharsky, G. J. Miller, and K. A. Gschneidner, Jr., Phys. Rev. B 69, 144102 (2004).

${ }^{15}$ C. Magen, L. Morellón, Z. Arnold, P. A. Algarabel, C. Ritter, M. R. Ibarra, J. Kamarad, A. O. Tsokol, K. A. Gschneidner, Jr., and V. K. Pecharsky, Phys. Rev. B 74, 134427 (2006).
${ }^{16}$ N. Marcano, P. A. Algarabel, J. Rodríguez Fernández, C. Magén, L. Morellón, Niraj K. Singh, D. L. Schlagel, K. A. Gschneidner, Jr., V. K. Pecharsky and M. R. Ibarra, Phys. Rev. B 85, 024408 (2012).

${ }^{17}$ Z. Arnold, C. Magén, L. Morellón, P. A. Algarabel, J. Kamarad, M. R. Ibarra, V. K. Pecharsky, and K. A. Gschneidner, Jr., Phys. Rev. B 79, 144430 (2009).

${ }^{18}$ L. Morellón, C. Ritter, C. Magén, P. A. Algarabel, and M. R. Ibarra, Phys. Rev. B 68, 024417 (2003).

${ }^{19}$ L. Morellón, Z. Arnold, C. Magen, C. Ritter, O. Prokhnenko, Y. Skorokhod, P. A. Algarabel, M. R. Ibarra, and J. Kamarad, Phys. Rev. Lett. 93, 137201 (2004).

${ }^{20}$ K. A. Gschneidner, Jr., V. K. Pecharsky, A. O. Pecharsky, V. V. Ivtchenko, and E. M. Levin, J. Alloys Compd 303-304, 214 (2000).

${ }^{21}$ R. Nirmala, Ya. Mudryk, V. K. Pecharsky, and K. A. Gschneidner, Jr., Phys. Rev. B 76, 104417 (2007).

${ }^{22}$ R. Nirmala, Durga Paudyal, V. K. Pecharsky, K. A. Gschneidner, Jr. and A. K. Nigam, J. Appl. Phys. 109, 07A923 (2011).

${ }^{23}$ Materials Preparation Center, The Ames Laboratory, US DOE, Ames, Iowa, USA, http://www.mpc.ameslab.gov.

${ }^{24} \mathrm{http}: / /$ www.easylab.co.uk

${ }^{25}$ Ivtchenko et al., in Advances in Cryogenic Engineering (Materials), edited by U. B. Balachandran et al., (Kluwer Academic/Plenum Publishers, New York, 2000), Vol. 46.

${ }^{26}$ L. Morellón, Z. Arnol, P. A. Algarabel, C. Magén, M. R. Ibarra, and Y. Skorokhod, J. Phys.: Condens. Matter 16, 1623 (2004).

${ }^{27}$ C. Magén, L. Morellón, P. A. Algarabel, M. R. Ibarra, C. Ritter, A. O. Pecharsky, K. A. Gschneidner Jr., and V. K. Pecharsky, Phys. Rev. B 70, 224429 (2004).

${ }^{28}$ Y. Imry and M. Wortis, Phys. Rev. B 19, 3580 (1979).

${ }^{29}$ Z. W. Ouyang, V. K. Pecharsky, K. A. Gschneidner, Jr., D. L. Schlagel, and T. A. Lograsso, Phys. Rev. B. 74, 094404 (2006).

${ }^{30}$ R. B. Griffiths, Phys. Rev. Lett. 23, 17 (1969).

${ }^{31}$ A. H. Castro Neto, G. Castilla, and B. A. Jones, Phys. Rev. Lett. 81, 3531 (1998).

${ }^{32}$ C. Magén, C. Ritter, L. Morellón, P. A. Algarabel, M. R. Ibarra, A. O. Tsokol, K. A. Gschneidner Jr., and V. K. Pecharsky, Phys. Rev. B 74, 174413 (2006). 